\title{
STABILITY PROPERTY OF MÖBIUS MAPPINGS
}

\author{
T. IWANIEC
}

\begin{abstract}
Let $F$ be an arbitrary class of continuous mappings acting and ranging on domains in $\mathbf{R}^{n}$ which is invariant under similarity transformations of $\mathbf{R}^{n}$ and the restriction of a map to any subdomain. The class Möb of Möbius mappings acting in $\mathbf{R}^{n}$ is of particular interest. Assume that the class $F$ is " $c$-uniformly close" to Möb. Then we show that any map in $F$ is either constant or a local quasiconformal homeomorphism. As a corollary we obtain a distinctly elementary proof of the Local Injectivity Theorem for quasiregular mappings.
\end{abstract}

Introduction. A sense preserving diffeomorphism $f: \Omega \rightarrow \mathbf{R}^{n}$, where $\Omega$ is a domain in $\mathbf{R}^{n}$, is said to be conformal if its Jacobian matrix $D f(x)$ at every point $x \in \Omega$ is a similarity transformation of $\mathbf{R}^{n}$. This means that $f$ satisfies a nonlinear system of p.d.e.

$$
(D f(x))^{T}(D f(x))=J(x, f)^{2 / n} I
$$

where $J(x, f)$ denotes the Jacobian determinant and $I$ is the identity matrix.

For $n=2$ this matrix equation coincides with the Cauchy-Riemann system. For this reason the solutions of (1) should be viewed as $n$-dimensional analogues of the analytic functions of one complex variable. For $n \geq 3$, however, system (1) is overdetermined and in this setting it behaves more like an ordinary differential equation system than like a typical partial differential equation. That is why the family of all solutions of (1) depends on a finite number of real parameters. More precisely, seeking solutions in the Sobolev space $W_{n, \text { loc }}^{1}(\Omega), n \geq 3$, one finds

THEOREM. Every nonconsant solution of (1) is a Möbius transformation.

Recall that a Möbius transformation is any finite composition of reflections in spheres or planes.

This result, referred to as Liouville's Theorem, was first proved without any a priori regularity hypothesis by F. W. Gehring [2] for locally injective mappings. The general case was treated by J. Rešetniak [5]. A purely analytic proof, independent of any result of quasiconformal mapping theory, was given by B. Bojarski and the author $[\mathbf{1}]$ in studying a local uniqueness criterion for system (1), the so-called Point Cauchy Problem.

There have been many efforts to generalize the theory of analytic functions to higher dimensions. The theory of holomorphic functions of several complex variables offers one possibility. Other efforts resulted in the concept of a quasiregular map (q.r. map).

Received by the editors December 4, 1984 and, in revised form, March 19, 1986.

1980 Mathematics Subject Classification (1985 Revision). Primary 30C60; Secondary 50Dxx.

This research was done while the author was visiting the University of Texas at Austin in 1984-1985. 
Definition (J. REŠEtNiAK). A map $f: \Omega \rightarrow \mathbf{R}^{n}$ is said to be K-quasiregular, $1 \leq K<\infty$, if $f \in W_{n, \mathrm{loc}}^{1}(\Omega)$ and if

$$
|D f(x)|^{n} \leq K J(x, f), \quad \text { a.e. }
$$

The smallest $K$ for which (2) holds is referred to as the distortion of $f$. In what follows the distortion is treated as a parameter classifying all quasiregular mappings. The case $K=1$ is extremal and inequality (2) reduces to the $n$-dimensional Cauchy-Riemann system (1). Generally, for $n \geq 3$, the number $K-1$ provides a measure of the "nearness" of the $K$-q.r. map to a Möbius map. We will not discuss q.r. mappings in detail but a few points deserve clarification.

It is natural that in the study of q.r. mappings one must use the technique of p.d.e., especially those methods concerning nonlinear degenerate elliptic equations. The early papers by J. Resetniak opened the way to the treatment of q.r. maps using topological arguments, a program carried out by O. Martio, S. Rickman, and J. Väisälä. Employing both analytical and geometric properties of a q.r. map V. M. Goldstein [3], O. Martio, S. Rickman, and J. Väisälä [4] proved the following.

LOCAL INJECTIVITY THEOREM. Every $K$-q.r. map in a domain of $\mathbf{R}^{n}, n \geq 3$, with $K$ sufficiently close to 1 is either constant or a local homeomorphism.

Thus topologically nontrivial q.r mappings in higher dimensions exist only in the presence of large distortion. As a matter of fact this theorem is a direct consequence of the Liouville Theorem and stability properties of Möbius mappings. There is very little we need to know about q.r. mappings, essentially the compactness property of quasiregular mapping families. Let us frame this subject in greater generality to make the arguments used, as well as the intrinsic nature of the result obtained, more apparent. For this, instead of q.r. maps, we consider a one-parameter family $\mathcal{F}=\left\{F_{t}\right\}_{t \geq 0}$, where the $F_{t}$ are fairly general classes of mappings acting on domains in $\mathbf{R}^{n}, n \geq 2$. It will be reasonable to assume that the $F_{t}$ are invariant under restrictions and elementary transformations of $\mathbf{R}^{n}$ (see conditions (i)-(ii)). We require that $F_{t}$ approach the Möbius class as $t$ tends to zero, in the sense of Definition 1. Under these few assumptions we are led to the conclusion: For small $t$ every map $f \in F_{t}$ must be constant or a local quasiconformal homeomorphism. Moreover we obtain a uniform estimate for the linear dilatation of mappings in $F_{t}$ (see Theorem 1). Hence, one sees that the only reasonable mappings close to Möbius are those which are $(1+\varepsilon)$-quasiconformal.

Theorem 1 applies to the $K$-q.r. families $(t=K-1$ e.g.) giving a new proof of the Local Injectivity Theoerem. The method presented here is distinctly elementary, so avoids appealing to a number of nontrivial results concerning q.r. mappings. In particular, the openness, the discreteness, and the structure of the branch set of a q.r. map are not utilized. Note that an estimate for the "radius of injectivity" is also provided by Corollary 1 .

For a detailed discussion of stability in the Liouville Theorem in more than two variables and of related topics we refer the reader to the recent book by J. Rešetniak [6]. We believe that some of the ideas described below are pertinent in other contexts, for instance, in the study of homogeneous systems of p.d.e.

ACKNOWLedgment. I wish to express my sincere thanks to Bruce Palka for many helpful suggestions and for carefully reading the manuscript. I also wish to 
thank him for directing a very inspirational seminar on complex analysis at The University of Texas at Austin, where I was a visitor in 1984-1985.

1. $c$-uniform variation of a mapping set. Given Euclidean spaces $\mathbf{R}^{n}$ and $\mathbf{R}^{m}$, a mapping set $F$ is any collection of continuous mappings $f: \Omega \rightarrow \mathbf{R}^{m}$, where $\Omega$ is a (variable) open subset of $\mathbf{R}^{n}$. Whenever we wish to specify the domain, say $\Omega$, of a map $f$ in $F$, we write $f \in F(\Omega)$.

Throughout this paper the mapping sets are tacitly assumed to be:

(i) closed under localization: $f \in F(\Omega)$ implies $f_{\mid U} \in F(U)$ for any subdomain $U \subset \Omega$, and

(ii) translation and dilation invariant: if the map $y=f(x)$ belongs to $F$, so also do the mappings $y=f\left(x+x_{0}\right), y=f(x)+y_{0}, y=f(\lambda x)$, and $y=\lambda f(x)$, where $x_{0} \in \mathbf{R}^{n}, y_{0} \in \mathbf{R}^{m}$, and $\lambda$ is a positive scalar.

If $f: \Omega \rightarrow \mathbf{R}^{m}$ is bounded we denote

$$
|f|_{\Omega}=\sup _{x \in \Omega}|f(x)| .
$$

A sequence $\left\{f_{k}\right\}_{k=1,2, \ldots}, f_{k}: \Omega \rightarrow \mathbf{R}^{m}$, is said to be bounded if $\left|f_{k}\right|_{\Omega} \leq M<\infty$ for $k=1,2, \ldots$ It is said to converge $c$-uniformly to a map $f: \Omega \rightarrow \mathbf{R}^{m}$ if $\lim \left|f_{k}-f\right|_{G}=0$ for every compact $G \subset \Omega$.

DEFINITION 1. Let $F$ be a mapping set. By a c-uniform variation of $F$ we mean $a$ one-parameter family $\mathcal{F}=\left\{F_{t}\right\}_{t \geq 0}$ of mapping sets $F_{t}$ subject to the conditions

$(*) F_{0}=F$;

(**) from any bounded sequence $\left\{f_{k}\right\}$ with $f_{k} \in F_{t_{k}}(\Omega)$ and $t_{k} \rightarrow 0$, it is possible to extract a subsequence converging c-uniformly to a map in $F(\Omega)$.

Such variation families typically occur in the study of homogeneous systems of p.d.e., where the $c$-uniform convergence follows from expedient a priori estimates.

In what follows the results depend on a function $\tau=\tau(t), t \geq 0$, associated with a given variation $\mathcal{F}=\left\{F_{t}\right\}_{t \geq 0}$ of $F$.

Let $\mathbf{B}$ be the unit ball in $\mathbf{R}^{n}$. Introduce the following distance function:

For $t \geq 0$, define

$$
d(f, g)=\sup _{x \in \mathbf{B}}(1-|x|)|f(x)-g(x)| .
$$

$$
\tau(t)=\sup _{\substack{f \in F_{s}(\mathbf{B}) \\ \boldsymbol{s} \leq t \leq t \\|f|_{\mathbf{B}} \leq \mathbf{1}}} \min _{\substack{\phi \in F(\mathbf{B}) \\ \phi(0)=f(0)}} d(f, \phi) .
$$

Notice that the map $\phi \in F(\mathbf{B}), \phi(0)=f(0)$, which minimizes $d(f, \phi)$ exists by condition $(* *)$ in Definition 1.

Clearly, $\tau(t)$ is an increasing function and $\tau(0)=0$. More importantly

$$
\lim _{t \rightarrow 0} \tau(t)=0 \text {. }
$$

To prove this assume to the contrary that $\tau\left(t_{k}\right)>\tau_{0}>0$ for some sequence $t_{k} \rightarrow 0$. This means there exist mappings $f_{k}, k=1,2, \ldots$, such that

$$
f_{k} \in F_{s_{k}}(\mathbf{B}), \quad 0 \leq s_{k} \leq t_{k}, \quad\left|f_{k}\right|_{\mathbf{B}} \leq 1,
$$

and

$$
d\left(f_{k}, \phi\right)>\tau_{0}
$$

for each $\phi \in F(\mathbf{B})$ normalized by $\phi(0)=f_{k}(0)$. 
By condition $(* *)$ a subsequence $\left\{f_{k_{\nu}}\right\}, \nu=1,2, \ldots$, can be selected which converges $c$-uniformly on $\mathbf{B}$ to a map $f \in F(\mathbf{B})$. A contradiction arises from substituting $\phi=f-f(0)+f_{k_{\nu}}(0)$ into (5) and letting the subindex $\nu$ go to infinity. Now the definition of $\tau(t)$ gives

LEMMA 1. Suppose $f \in F_{t}(B)$, where $B=B(a, r)$ is an arbitrary ball in $\mathbf{R}^{n}$. There exists $\phi \in F(B)$ such that

$$
\phi(a)=f(a), \quad|f-\phi|_{\sigma B} \leq \frac{\tau}{1-\sigma}|f|_{B}, \quad \tau=\tau(t),
$$

for every $\sigma \in[0,1)$.

(Here and in what follows the notation $\sigma B$ is used for the ball $B(a, \sigma r)$.)

PROOF. With the aid of elementary transformations of variables of $\mathbf{R}^{n}$ and $\mathbf{R}^{m}$ the problem reduces to the unit ball $\mathbf{B}$ and $|f|_{\mathbf{B}}=1$. (The case $|f|_{B}=0$ is trivial.) By (3) we find a map $\phi \in F(\mathbf{B})$ such that $\phi(0)=f(0)$ and $d(f, \phi) \leq \tau$. This implies (6), since $1-|x| \geq 1-\sigma$ in the ball $\sigma \mathbf{B}$.

Since it is an immediate consequence of simple compactness arguments, Lemma 1 might be viewed as a very crude stability result. We shall see, however, that this lemma is strong enough to allow for useful applications. Owing to the geometry of balls in $\mathbf{R}^{n}$, various properties of mappings in $F$ can be carried over to the mappings in $F_{t}$ for small $t$. Note that all estimates occurring in the sequel will be effective, apart from the presence of the function $\tau(t)$.

2. The Möbius mappings. For notational convenience it is advantageous to include constant mappings in the Möbius mapping set. Therefore a map $\phi: \Omega \rightarrow$ $\mathbf{R}^{n}, \Omega \subset \mathbf{R}^{n}, n \geq 2$, will be called Möbius if it is either constant or the restriction to $\Omega$ of a Möbius transformation acting in $\overline{\mathbf{R}^{n}}$. We should point out that the notation $\phi \in \operatorname{Möb}(\Omega)$ includes the assumption that the pole $p=\phi^{-1}(\infty)$ does not lie in $\Omega$. The complete Möbius mapping set, where the domain of a map is left unspecified, will be denoted by

$$
\operatorname{Möb}=\bigcup_{\Omega \subset \mathbf{R}^{n}} \operatorname{Möb}(\Omega) .
$$

We shall use the following characteristic property of a Möbius transformation. Given four distinct points $x, y, u, v$ in $\mathbf{R}^{n}$, the cross-ratio of these points is

$$
\frac{|x-u||y-v|}{|x-y||u-v|}
$$

Every Möbius transformation $\phi$ preserves cross-ratios. Using this invariance of cross-ratios twice we obtain a very useful formula:

$$
|x-p||y-p| \frac{|\phi(x)-\phi(y)|}{|x-y|}=|u-p||v-p| \frac{|\phi(u)-\phi(v)|}{|u-v|}
$$

where $p=\phi^{-1}(\infty)$ is the pole of $\phi$. Should $p=\infty$, the obvious reduction occurs in this identity, which also holds for a constant map.

The next two propositions should be interpreted as the distortion inequality and averaged Harnack estimate for Möbius mappings. 
Proposition 1. Let $B=B(a, r)$ and $\phi \in \operatorname{Möb}(B)$. Then

$$
\max _{|x-a| \leq \sigma r}|\phi(x)-\phi(a)| \leq \frac{1+\sigma}{1-\sigma} \min _{|x-a|=\sigma r}|\phi(x)-\phi(a)|
$$

for every $\sigma \in[0,1)$.

Proposition 2. Let $\phi \in \operatorname{Möb}(B), B=B(a, r)$, and let $0<\nu \leq \sigma<1$. Then

$$
|\phi|_{\sigma B} \leq \frac{\sigma-\nu^{2}}{\nu(1-\sigma)}|\phi|_{\nu B}
$$

ProOF. We may assume that $B$ is the unit ball $\mathbf{B}$ and that $\phi$ is nonconstant. In particular, $|p| \geq 1$ for $p=\phi^{-1}(\infty)$. Apply identity (7) to $y=v=0$ and to arbitrary $x$ and $u$ such that $|x|=|u|=\sigma$.

$$
|\phi(x)-\phi(0)|=\frac{|u-p|}{|x-p|}|\phi(u)-\phi(0)| .
$$

This, along with the elementary inequality

$$
\frac{|u-p|}{|x-p|} \leq \frac{|p|+\sigma}{|p|-\sigma} \leq \frac{1+\sigma}{1-\sigma}
$$

proves Proposition 1.

To prove Proposition 2 we let $w$ be an arbitrary point in the unit ball. We apply (7) to the quadruple

$$
(x, y, u, v)=\left(\sigma w, \nu w, \frac{\nu p}{|p|},-\frac{\nu p}{|p|}\right)
$$

to get

$$
\begin{aligned}
|\phi(\sigma w)-\phi(\nu w)| & =\frac{(\sigma-\nu)\left(|p|^{2}-\nu^{2}\right)|w|}{2 \nu|p-\sigma w||p-\nu w|}\left|\phi\left(\frac{\nu p}{|p|}\right)-\phi\left(-\frac{\nu p}{|p|}\right)\right| \\
& \leq \frac{(\sigma-\nu)(1+\nu)}{2 \nu(1-\sigma)} 2|\phi|_{\nu \mathbf{B}} .
\end{aligned}
$$

Since $\sigma w$ and $\nu w$ vary over the balls $\sigma \mathbf{B}$ and $\nu \mathbf{B}$ we conclude

$$
\begin{aligned}
|\phi|_{\sigma \mathbf{B}} & \leq\left(1+\frac{(\sigma-\nu)(1+\nu)}{\nu(1-\sigma)}\right)|\phi|_{\nu \mathbf{B}} \\
& =\frac{\sigma-\nu^{2}}{\nu(1-\sigma)}|\phi|_{\nu \mathbf{B}}
\end{aligned}
$$

This completes the proof of Proposition 2.

From now on $\mathcal{F}=\left\{F_{t}\right\}_{t \geq 0}$ will be a fixed $c$-uniform variation of the Möbius mapping set. Whenever we appeal to Lemma 1 we do so under the assumption that $n=m, F=\mathrm{Möb}$, and $\tau=\tau(t)$, the stability function associated with the variation $\mathcal{F}$. Our immediate goal is to carry over Propositions 1 and 2 to the classes $F_{t}$ for small $t$. We forgo possibly more sophisticated estimates in favor of simple arithmetic. 
3. Harnack's estimates. We begin with the following auxiliary proposition.

Proposition 3. Let $f \in F_{t}(\Omega)$. Then

$$
|f|_{4 B / 5} \leq 8|f|_{2 B / 5}+19 \tau|f|_{B}
$$

for every ball $B \subset \Omega$, where $\tau=\tau(t)$.

ProOF. Apply Lemma 1 with $\sigma=\frac{4}{5}$ and Proposition 2 with $\sigma=\frac{4}{5}, \nu=\frac{2}{5}$. Then by the triangle inequality

$$
\begin{aligned}
|f|_{4 B / 5} & \leq|\phi|_{4 B / 5}+|f-\phi|_{4 B / 5} \leq 8|\phi|_{2 B / 5}+5 \tau|f|_{B} \\
& \leq 8|f|_{2 B / 5}+8|f-\phi|_{2 B / 5}+5 \tau|f|_{B} .
\end{aligned}
$$

To estimate $|f-\phi|_{2 B / 5}$ apply Lemma 1 again, but with $\sigma=\frac{2}{5}$. This gives (10).

The next step is to eliminate the undesirable term $19 \tau|f|_{B}$ from (10). The following geometric lemma gives a clue to achieving this.

LEMMA 2. Let $f$ be bounded in $\Omega$ and satisfy

$$
2|f|_{4 B / 5} \leq M|f|_{2 / 5 B}+\frac{1}{M}|f|_{B}
$$

for every ball $B \subset \Omega$, where $M>1$ is a constant independent of the ball. Then $f$ satisfies Harnack's inequality:

$$
|f|_{\left(1-q^{l}\right) B} \leq M^{l-1}|f|_{(1-q) B}
$$

for every ball $B \subset \Omega$, where $\frac{1}{2} \leq q<1$ and $l=1,2, \ldots$.

ProOF. For notational simplicity we assume that $B$ in (12) is the unit ball $\mathbf{B}$. Fix $q \in\left[\frac{1}{2}, 1\right)$ and consider an ascending sequence of concentric balls

$$
B_{m}=\left(1-q^{m}\right) \mathbf{B}, \quad m=1,2, \ldots
$$

Note that

$$
1-q^{m}>2(1-q) q^{m-1}
$$

for $m=2,3, \ldots$ Now consider an arbitrary ball $B^{\prime} \subset B_{m}$ with radius $2(1-q) q^{m-1}$. Of course the collection of such balls $B^{\prime}$ cover $B_{m}$. On the other hand, one sees that

$$
\frac{1}{2} B^{\prime} \subset B_{m-1} \quad \text { and } \quad \frac{5}{4} B^{\prime} \subset B_{m+1}
$$

for $m=2,3, \ldots$ Applying (11) to the ball $\frac{5}{4} B^{\prime}$ gives

Hence

$$
2|f|_{B^{\prime}} \leq M|f|_{B^{\prime} / 2}+\frac{1}{M}|f|_{5 B^{\prime} / 4}
$$

or, equivalently

$$
2|f|_{B_{m}} \leq M|f|_{B_{m-1}}+\frac{1}{M}|f|_{B_{m+1}}
$$

$$
2 M^{-m}|f|_{B_{m}} \leq M^{-(m-1)}|f|_{B_{m-1}}+M^{-(m+1)}|f|_{B_{m+1}}
$$

for $m=2,3, \ldots$ Fix an integer $l \geq 2$. Sum (13) over $m \geq l$, change indices in the summation sign and cancel out those terms with $m \geq l+1$ as follows:

$$
2 \sum_{m=l}^{\infty} M^{-m}|f|_{B_{m}} \leq \sum_{m=l-1}^{\infty} M^{-m}|f|_{B_{m}}+\sum_{m=l+1}^{\infty} M^{-m}|f|_{B_{m}} .
$$

Hence $|f|_{B_{l}} \leq M|f|_{B_{l-1}}$ for $l=2,3, \ldots$ By iteration, $|f|_{B_{l}} \leq M^{l-1}|f|_{B_{1}}$, which obviously holds for $l=1$ as well. This completes the proof of the lemma.

Now we are ready to formulate Harnack's inequality for mappings in $F_{t}$. 
LEMMA 3. Let $f \in F_{t}(\Omega)$, where $t$ is small enough to satisfy $\tau=\tau(t) \leq 0.001$. Then for every ball $B \subset \Omega$

$$
|f|_{B / 2} \leq 2^{(4-4 \rho) / \rho}|f|_{\rho B}
$$

where $0<\rho \leq \frac{1}{2}$.

ProOF. It suffices to prove (14) when $f$ is bounded in $B$. The general case would follow by applying this particular case to any smaller concentric ball. Inequality (10) and $\tau \leq 0.001$ guarantee that the hypothesis of Lemma 2 is satisfied with $M=16$, from which we infer

$$
|f|_{\left(1-q^{l}\right) B} \leq 16^{l-1}|f|_{(1-q) B}
$$

Put $q=(1-\rho) \geq \frac{1}{2}$ and let $l$ be the integer such that $(1-\rho) / \rho \leq l<1 / \rho$. Bernoulli's inequality yields

$$
q^{l} \leq q^{(1-\rho) / \rho}=\left(1+\frac{1-q}{q}\right)^{-q /(1-q)} \leq(1+1)^{-1}=\frac{1}{2} .
$$

Hence $1-q^{l} \geq \frac{1}{2}$. This implies (14).

4. Distortion inequality and local injectivity. Proposition 1 can be carried over to the mappings in $F_{t}$ as follows.

LEMMA 4. Let $B=B(a, R)$ be a ball in $\mathbf{R}^{n}$ and let $f \in F_{t}(B)$, where $t$ is so small that $\tau=\tau(t) \leq 0.00001$. Then

$$
\max _{|x-a| \leq \rho R}|f(x)-f(a)| \leq\left(\frac{1+2 \rho}{1-2 \rho}+3 \cdot 2^{4 / \rho} \tau\right) \min _{|x-a|=\rho R}|f(x)-f(a)|
$$

for every $\rho \in\left(0, \frac{1}{4}\right]$.

PROOF. By elementary transformations this problem reduces to the case $a=0$ and $f(a)=0$. Applying Lemma 1 to the ball $B\left(0, \frac{1}{2} R\right)$ and $\sigma=2 \rho \leq \frac{1}{2}$ we find a map $\phi \in \operatorname{Möb}\left(\frac{1}{2} B\right)$ such that $\phi(0)=0$ and

$$
|f-\phi|_{\rho B} \leq 2 \tau|f|_{B / 2} \text {. }
$$

By Harnack's inequality (see Lemma 3 ) this gives

$$
|f-\phi|_{\rho B} \leq 2^{4 / \rho-3} \tau|f|_{\rho B}
$$

On the other hand we have the following distortion estimate for the Möbius map $\phi$ :

$$
\max _{|x| \leq \rho R}|\phi(x)| \leq \frac{1+2 \rho}{1-2 \rho} \min _{|x|=\rho R}|\phi(x)| .
$$

This follows from Proposition 1 applied to the ball $B\left(0, \frac{1}{2} R\right)$ and $\sigma=2 \rho$. The rest of the proof is a matter of applying the triangle inequality,

$$
\begin{aligned}
|f|_{\rho B} & \leq|\phi|_{\rho B}+|f-\phi|_{\rho B} \leq \frac{1+2 \rho}{1-2 \rho} \min _{|x|=\rho R}|\phi(x)|+|f-\phi|_{\rho B} \\
& \leq \frac{1+2 \rho}{1-2 \rho} \min _{|x|=\rho R}|f(x)|+\frac{1+2 \rho}{1-2 \rho}|f-\phi|_{\rho B}+|f-\phi|_{\rho B} \\
& =\frac{1+2 \rho}{1-2 \rho} \min _{|x|=\rho R}|f(x)|+\frac{2}{1-2 \rho}|f-\phi|_{\rho B} .
\end{aligned}
$$


Hence

$$
|f|_{\rho B} \leq \frac{1+2 \rho}{1-2 \rho} \min _{|x|=\rho R}|f(x)|+2^{4 / \rho-1} \tau|f|_{\rho B} .
$$

In particular for $\rho=\frac{1}{4}$ we have

$$
|f|_{B / 4} \leq 3 \min _{|x|=R / 4}|f(x)|+0.4|f|_{B / 4}
$$

and so

$$
|f|_{B / 4} \leq 5 \min _{|x|=R / 4}|f(x)|
$$

Notice that the last inequality holds for any concentric ball smaller than $B$ as well, giving

$$
|f|_{\rho B} \leq 5 \min _{|x|=\rho B}|f(x)|
$$

for every $\rho \in\left(0, \frac{1}{4}\right]$. Returning to $(16)$ we finally arrive at

$$
\max _{|x| \leq \rho R}|f(x)| \leq\left(\frac{1+2 \rho}{1-2 \rho}+3 \cdot 2^{4 / \rho} \tau\right) \min _{|x|=\rho R}|f(x)| .
$$

COROLLARY 1. Let $\Omega \subset \mathbf{R}^{n}$ be connected and let $f$ be an arbitrary nonconstant map in $F_{t}(\Omega)$, where $t$ is so small that $\tau(t) \leq 0.00001$. Then $f$ is injective in every ball $B$ such that $9 B \subset \Omega$.

PROOF. Suppose that there are distinct points $a, b \in B$, where $9 B \subset \Omega$, such that $f(a)=f(b)$. It is geometrically clear that $4|b-a| \leq \operatorname{dist}(a, \partial \Omega)$. Applying (15) to $\rho=\frac{1}{4}$ and $R=4|b-a|$ yields

$$
\max _{|x-a| \leq R}|f(x)-f(a)| \leq 5|f(b)-f(a)|=0 .
$$

This shows that $f$ is constant on the ball $B\left(a, \frac{1}{4} R\right)$. By repitition of this argument we find that $f$ is constant on the whole domain $\Omega$, contrary to hypothesis.

Summary. To state the conclusion we need finally the following metric definition of a quasiconformal map.

Definition (F. W. GeHRING). A local homeomorphism $f: \Omega \rightarrow \mathbf{R}^{n}, \Omega \subset$ $\mathbf{R}^{n}$, is said to be $K$-quasiconformal, $1 \leq K<\infty$, if

$$
\varlimsup_{r \rightarrow 0} \frac{\max _{|x-a|=r}|f(x)-f(a)|}{\min _{|x-a|=r}|f(x)-f(a)|} \leq \mathcal{K}
$$

for each $a \in \Omega$. The smallest $\mathcal{K}$ for which (17) holds is called the linear dilatation of $f$. We denote it by $\mathcal{K}_{f}$.

It follows from Lemma 4 that every nonconstant map $f \in F_{t}$, where $\tau(t) \leq$ 0.00001 , is locally quasiconformal. Its linear dilatation can be estimated uniformly by means of the parameter $t$.

Denote by

$$
\mathcal{K}(t)=\sup \left\{K_{f} ; f \in F_{t} \text { and } f \text { is nonconstant }\right\}
$$

for $t$ such that $\tau(t) \leq 0.00001$. Then by (15)

$$
\mathcal{K}(t) \leq \inf \left\{\frac{1+2 \rho}{1-2 \rho}+3 \cdot 2^{4 / \rho} \cdot \tau(t) ; 0<\rho \leq \frac{1}{4}\right\}
$$

which shows that $\mathcal{K}(t)=1+o(1)$ as $t$ goes to 0 . 
THEOREM 1. Consider a c-uniform variation $\mathcal{F}=\left\{F_{t}\right\}_{t \geq 0}$ of the Möbius mapping set in $\mathbf{R}^{n}, n \geq 2$. For sufficiently small $t$ the following is true:

(a) every nonconstant map $f$ in $F_{t}$ is a locally quasiconformal map,

(b) there exists $\mathcal{K}(t)=1+o(1)$ such that $\mathcal{K}_{f} \leq \mathcal{K}(t)$ for every nonconstant $f \in F_{t}$.

\section{REFERENCES}

1. B. Bojarski and T. Iwaniec, Another approach to Liouville theorem, Math. Nachr. 107 (1982), 253-262.

2. F. W. Gehring, Rings and quasiconformal mappings in space, Proc. Nat. Acad. Sci. U.S.A. 47 (1961), 98-105.

3. V. M. Goldstein, On the behaviour of mappings with bounded distortion with distortion coefficient close to unity, Siberian Math. J. 12 (1971), 1250-1258.

4. O. Martio, S. Rickman, and J. Väisälä, Topological and metric properties of quasiregular mappings, Ann. Acad. Sci. Fenn. Ser. A I Math. 488 (1971), 1-31.

5. J. Rešetniak, Liouville conformal mapping theorem under minimal regularity hypothesis, Siberian Math. J. 8 (1967), 835-840.

6. __ Stability theorems in geometry and analysis, "Nauka", Novosibirsk, 1982. (Russian)

Department of Mathematics, University of Texas, Austin, TeXas 78713

Current address: Department of Mathematics, Syracuse University, Syracuse, New York 13210 